\title{
How Personification and Interactivity Influence Stress-Related Disclosures to Conversational Agents
}

\author{
Shruti Sannon \\ Brett Stoll \\ Dominic DiFranzo \\ Malte Jung \\ Natalya N. Bazarova \\ Cornell University \\ Ithaca, NY, USA \\ $\{$ ss3464, bas364, djd274, \\ mfj28, nnb8\}@cornell.edu
}

Permission to make digital or hard copies of part or all of this work for personal or classroom use is granted without fee provided that copies are not made or distributed for profit or commercial advantage and that copies bear this notice and the full citation on the first page. Copyrights for third-party components of this work must be honored. For all other uses, contact the Owner/Author.

CSCW '18 Companion, November 3-7, 2018, Jersey City, NJ, USA C) 2018 Copyright is held by the owner/author(s).

ACM ISBN 978-1-4503-6018-0/18/11.

https://doi.org/10.1145/3272973.3274076

\begin{abstract}
In this exploratory study, we examine how personification and interactivity may influence people's disclosures around sensitive topics, such as psychological stressors. Participants $(N=441)$ shared a recent stressful experience with one of three agent interfaces: 1) a non-interactive, non-personified survey, 2) an interactive, non-personified chatbot, and 3) an interactive, personified chatbot. We coded these responses to examine how agent type influenced the nature of the stressor disclosed, and the intimacy and amount of disclosure. Participants discussed fewer homelife related stressors, but more finance-related stressors and more chronic stressors overall with the personified chatbot than the other two agents. The personified chatbot was also twice as likely as the other agents to receive disclosures that contained very little detail. We discuss the role played by personification and interactivity in interactions with conversational agents, and implications for design.
\end{abstract}

\section{Author Keywords}

Conversational agents; disclosure; stress; personification; interactivity. 


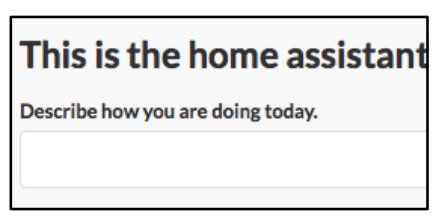

Figure 1. Survey

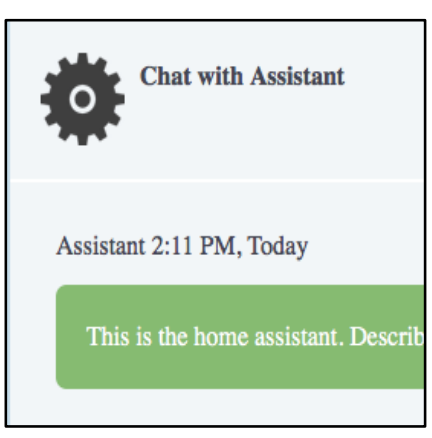

Figure 2. Non-Personified Chatbot

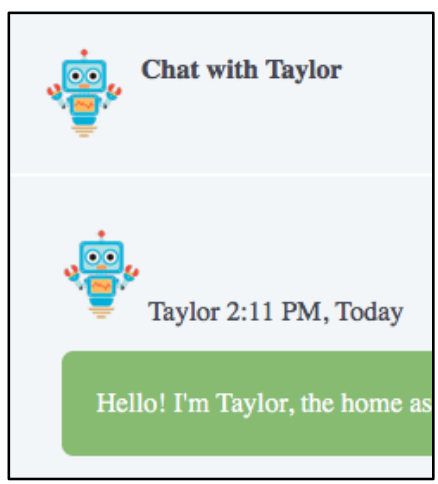

Figure 3. Personified Chatbot

\section{Introduction}

Conversational agents are increasingly becoming the primary interface for many systems, including those used for mental health, well-being, and stress management [4]. These agents vary in form, degree of artificial intelligence, and function. As part of these services, users often share personal or sensitive information with agents that play the role of a companion, coach, or helper.

As conversational agents become more ubiquitous in the domain of health, we expect the amount of sensitive disclosures with technology to increase. However, the influence of agent characteristics on users' disclosures about sensitive information, such as psychological stressors, is not fully understood. Are people more inclined to share sensitive, stressful experiences with conversational agents compared to surveys, and how do agent characteristics influence such disclosures? To address this question, this exploratory study examines interactions between online participants and one of three agent interfaces that vary in their levels of interactivity and personification.

Previous work on human-agent interaction has found that conversational agent characteristics impact people's perceptions and conversations. In general, conversational agents, compared to human partners, increase human willingness to disclose information [6], potentially due to lower concerns of evaluation and impression management around agents. However, people also send shorter, less vocabulary-rich messages to agents than to humans [2]. Agent personalities also impact disclosure: serious and assertive agents can engender greater disclosure than warm, cheerful ones [5]. Further research is needed to parse which anthropomorphizing, or human-like, characteristics of agents encourage or inhibit interpersonal disclosure about sensitive topics.

Anthropomorphism has two broad components: personification and interactivity. Personification refers to the agent's human-like cues (e.g., name, physical appearance, personality). Interactivity refers to the agent's communication and ability to converse, such that messages from the agent are perceived as relevant to and contingent upon human input [7]. Previous work has examined anthropomorphic versus nonanthropomorphic agents, but has not directly addressed how interactivity and personification shape stressrelated disclosures. As these agents become more common, understanding how these factors influence disclosure, particularly around sensitive topics, is important. Thus our research question:

RQ: How do the personification and interactivity of online interfaces influence users' disclosures about recent stressful events, in terms of a) the nature of the stressful event disclosed, and b) the intimacy and amount of information disclosed?

\section{Methods}

Data Collection

We recruited participants on Amazon Mechanical Turk $(N=467)$; participants were told they were testing a prototype of an online home assistant. Participants interacted with either 1) a non-interactive, nonpersonified Survey (baseline condition), 2) an interactive Non-personified Chatbot, or 3) an interactive Personified Chatbot. Participants were told that to receive personalized service, the assistant would ask them several questions. The agents asked them 


\section{Examples of \\ Participants' \\ Disclosures by Code}

Chronic: "I've been dealing with diabetes"

Non-Chronic: "I had a big presentation at work last week"

More detailed: "I've been really stressed out lately over my daughter moving out of the house with her boyfriend. She's never lived on her own before and I'm really worried that she'll need to come back."

Less detailed: "Money."

Home-related: "My

apartment flooded and my kid's bedroom was ruined"

Finance-related: "The rent in my apartment was just raised and I'm worried I won't be able to keep up with payments" about their interests, followed by an open-ended question about a recent stressor. The Survey and Nonpersonified Chatbot used identical language and only differed in interactivity: the survey was a static webform while the chatbot used a chat window. The two chatbots provided the same level of interactivity but differed in personification: the Personified Chatbot had a unisex name, picture, and used more conversational language. Pictures of each agent and example utterances are found in Figures 1-3.

\section{Content Analysis}

Two coders coded responses to the question about a stressful experience, and we compared these by condition (Survey, Non-Personified Chatbot, Personified Chatbot). 441 responses were coded, with 26 cases $(5.6 \%)$ removed for being nonsensical or irrelevant.

Nature of Stressful Event Disclosure. We coded responses according to the Inventory of Stressful Events [1], noting the topic of the event (homelife, work, finances, relationships, health, legal, education, misc.) $(\kappa=.87)$ and its ongoing difficulty (temporary, chronic, uncodable, or non-event) $(\kappa=.77)$.

Intimacy and Amount of Disclosure. We also coded responses for the intimacy of the disclosure using a 3pt scale $(1=$ relatively public or impersonal to $3=$ very private or personal), as in [3]. The amount of details in each disclosure was coded on a 3-pt scale ( $1=$ very little detail to $3=a$ lot of detail). Due to skewed distribution, we dichotomized this variable into "fewer details" and "more details". Intercoder reliability was acceptable for both intimacy $(\kappa=.71)$ and amount of details $(\kappa=.79)$.

\section{Results}

Nature of Stressful Event

A Chi-square test of independence indicated that the topics of participants' stressful experiences varied by agent type, $X 2(14)=27.54, p<.05$. We used post-hoc pairwise comparisons with Bonferroni corrections to examine specific differences, and found that only the homelife and finance topics varied by agent type (see Table 1). Specifically, the Personified Chatbot received fewer disclosures about homelife than the other agents $(x 2(1)=6.71, p<.01$, odds ratio $=0.53)$, and more disclosures about finances than the other agents $(x 2(1)=6.56, p<.01$, odds ratio $=1.82)$.

132 responses provided too little information to infer whether the stressor was chronic; we analyzed the remaining 309 responses. The Personified Chatbot received significantly more disclosures about chronic stressors than the other agents, $X 2(1)=12.02, p<.001$, odds ratio $=2.95$; there was no difference between the Non-personified Chatbot and the Survey (see Table 2).

Intimacy and Amount of Disclosure

There was no significant difference in the intimacy of disclosures based on agent type, $x 2(4)=7.82, p>.05$. In terms of the detail provided in disclosures (see Table 3 ), there was no significant difference between the level of detail disclosed to the Survey and the Nonpersonified Chatbot, suggesting that interactivity alone did not impact detail in disclosures. However, the Personified Chatbot was more than twice as likely as the other agents to receive responses that contained very little detail, suggesting that personification is the driving factor in the difference in disclosures, $x 2(1)=15.19, p<.001$, odds ratio $=2.81$. 


\begin{tabular}{r|rr} 
& Home & Finance \\
\hline $\begin{array}{r}\text { Survey } \\
\text { Non- }\end{array}$ & $33 \%$ & $19 \%$ \\
personified & $28 \%$ & $22 \%$ \\
Personified & $19 \%$ & $32 \%$
\end{tabular}

Table 1: Differences in disclosure about home or finance-related stressors by agent condition $(n=309)$

\begin{tabular}{r|rr}
\multicolumn{2}{c}{ Chronic } & $\begin{array}{r}\text { Non- } \\
\text { chronic }\end{array}$ \\
\hline $\begin{array}{r}\text { Non- } \\
\text { Norvey }\end{array}$ & $13 \%$ & $87 \%$ \\
personified & $18 \%$ & $82 \%$ \\
Personified & $35 \%$ & $65 \%$
\end{tabular}

Table 2: Whether the stressor disclosed poses ongoing difficulty, by agent condition $(n=309)$

\begin{tabular}{r|rr} 
& $\begin{array}{r}\text { Fewer } \\
\text { Details }\end{array}$ & $\begin{array}{r}\text { More } \\
\text { Details }\end{array}$ \\
\hline $\begin{array}{r}\text { Survey } \\
\text { Non- }\end{array}$ & $11 \%$ & $89 \%$ \\
personified & $12 \%$ & $88 \%$ \\
Personified & $27 \%$ & $73 \%$
\end{tabular}

Table 3: The amount of information and context in disclosures, by agent condition $(n=441)$

\section{Discussion}

Findings show that agent personification was a stronger indicator of both the nature and amount of disclosure than interactivity alone, such that the Personified chatbot elicited fewer detailed disclosures of stressors and more chronic stress events overall. Moreover, participants shared fewer homelife issues but more financial stressors with the Personified chatbot.

In [6], they argue that people may make limited disclosures to agents versus humans due to impression management concerns. Similar concerns of interpersonal evaluation may be triggered by a personified versus non-personified chatbot, and may explain why participants were less likely to share homelife stressors-that may be more sensitive-with the Personified Chatbot. In contrast, limited disclosure about finance (e.g., "money", "doing my taxes") may be seen by participants as less interpersonally intimate or risky compared to other stressful topics.

The personification of the chatbot might also set different expectations about the agent's expertise and ability to address certain stressful topics. This could influence which types of stressful events participants chose to disclose. People may also view a personified chatbot as less trustworthy in handling sensitive homelife information.

These findings suggest careful consideration of how agents are designed for interfaces geared toward sensitive disclosures. For discussions of stressful events, having a simpler, less personified agent may be better to encourage in-depth sensitive disclosures from users. Future work should continue to look at the nuances of interactivity and personification, and how each might affect sensitive disclosures differently.

\section{Acknowledgements}

We thank the National Science Foundation (award \#1405634), and our undergraduate RAs, Brenna

Garcia, Colton Zuvich, and Erin Chen, for their support.

\section{References}

1. David M. Almeida, Elaine Wethington, and Ronald C. Kessler. 2002. The Daily Inventory of Stressful Events: An interview-based approach for measuring daily stressors. Assessment.

2. Hill, Jennifer, W. Randolph Ford, and Ingrid G. Farreras. 2015. Real conversations with artificial intelligence: A comparison between human-human online conversations and human-chatbot conversations. Computers in Human Behavior.

3. Crystal Jiang, Natalya N. Bazarova, and Jeffrey T. Hancock. 2013. From perception to behavior: Disclosure reciprocity and the intensification of intimacy in computer-mediated communication. Communication Research.

4. Seung-A. A. Jin. 2010. The effects of incorporating a virtual agent in a computer-aided test designed for stress management education: The mediating role of enjoyment. Computers in Human Behavior.

5. Jingyi Li, Michelle X. Zhou, Huahai Yang, and Gloria Mark. 2017. Confiding in and Listening to Virtual Agents: The Effect of Personality. In Proc of IUI.

6. Gale M. Lucas et al. 2014. It's only a computer: Virtual humans increase willingness to disclose. Computers in Human Behavior.

7. Shyam Sundar. 2008. The MAIN model: A heuristic approach to understanding technology effects on credibility. Digital media, youth, and credibility. 